Marcel Helbig, Markus Konrad \& Rita Nikolai

\title{
Entwicklung der Schulinfrastruktur in Ostdeutschland und die Rolle privater Schulen
}

\section{Zusammenfassung}

Der Beitrag analysiert, wie sich das Schulnetz in den ostdeutschen Bundesländern in den letzten 25 Jahren entwickelt hat. Den Bundesländern gelang es in dieser Zeit relativ gut, eine Versorgung mit Grundschulen und Schulen mit gymnasialer Oberstufe abzusichern; weniger erfolgreich waren sie dagegen in der Versorgung mit Schulen ohne gymnasiale Oberstufe. Privatschulen spielten bei der räumlichen Grundversorgung mit Bildungseinrichtungen eine untergeordnete Rolle.

Schlüsselwörter: Schulinfrastruktur, Ostdeutschland, Dichte des Schulangebots, Schülerzahlen

\section{Development of the School Infrastructure in East Germany and the Role of Private Schools}

\begin{abstract}
The article analyzes the development of school sites in the last 25 years since the reunification. The East German Länder were successful in ensuring the coverage of primary schools and schools with an upper school form, but less of secondary schools without such forms. Private schools played a minor role for the basic and spatial supply of schools.
\end{abstract}

Keywords: school infrastructure, East Germany, coverage of schools, number of students 


\section{Einleitung ${ }^{1}$}

Nach der Wiedervereinigung mussten in den ostdeutschen Bundesländern aufgrund eines starken Rückgangs der Schülerzahlen viele öffentliche Schulstandorte geschlossen werden (z.B. Fickermann, Schulzeck \& Weishaupt, 2000; Weishaupt, 2018). Zurückgehende Schülerzahlen betreffen jedoch nicht nur die ostdeutschen Bundesländer, sondern abgeschwächt auch die westdeutschen Bundesländer. Sich verändernde Schülerzahlen bedürfen also weiterhin der steuernden Planung durch Ministerien und Kommunen. Bei ihrer Schulentwicklungsplanung müssen diese Akteure vielfältige Entscheidungsparameter berücksichtigen, zu denen vor dem Hintergrund der Ressourcenabschätzungen Fragen zur inhaltlichen Standortsicherung, zur Qualitätsentwicklung wie auch zur Sicherstellung eines Reservoirs von gut ausgebildeten Lehrkräften gehören. Wie Kommunen und Ministerien mit solchen Herausforderungen umgehen und zu deren Entscheidungsprozessen liegen bereits zahlreiche Analysen vor (z. B. Kann, 2017; Walde, 2019). Sie stehen deshalb auch nicht im Fokus der vorliegenden Analysen.

Wir wollen stattdessen im Kontext von Schulschließungen und Zusammenlegungen von Schulformen untersuchen, wie sich die räumliche Schulversorgung verändert hat und welche Rolle Privatschulen ${ }^{2}$ für Veränderungen der Schulinfrastruktur hatten. Im aktuellen Nationalen Bildungsbericht (Autorengruppe Bildungsberichterstattung, 2018, S. 88) wurde die Annahme formuliert, dass private Anbieter gerade im ländlichen Raum Ostdeutschlands Schulangebote aufrechterhalten haben. In den ostdeutschen Bundesländern wurden tatsächlich viele Privatschulen gerade im Grundschulbereich gegründet (Koinzer \& Mayer, 2015). Jedoch fehlt es bislang an Untersuchungen, welchen Beitrag Privatschulen für die Schulversorgung und den Erhalt einer wohnortnahen Beschulung in dünn besiedelten ländlichen Regionen leisten. In unserem Beitrag untersuchen wir folgende Fragestellungen:

1) Wie hat sich die Schulinfrastruktur in Ostdeutschland in den letzten 25 Jahren entwickelt?

2) Welche Rolle spielten dabei die Privatschulen?

3) Inwieweit haben schulrechtliche Vorgaben wie Mindestklassengrößen und Möglichkeiten zur Zusammenfassung von Schulformen die Schulversorgung beeinflusst?

1 Rita Nikolai wird durch ein Heisenberg-Stipendium der Deutschen Forschungsgemeinschaft gefördert (Förderkennzeichen: NI 1371/4-1). Wir danken Sophie Drebes und Martin Bücher für Unterstützung bei Recherche und Aufbereitung der Schullisten. Ebenso bedanken wir uns bei den Gutachter*innen für hilfreiche Anregungen.

2 Die Begriffe „private Schule“, „Privatschule“ sowie „Schule in freier Trägerschaft" verwenden wir synonym und schließen uns mit dem Begriff „Privatschule“ der Begrifflichkeit des Grundgesetzes an. Zudem beziehen wir uns auf die allgemeinbildenden und nicht auf die berufsbildenden und sonstigen Privatschulen. 
Inwieweit es den ostdeutschen Bundesländern gelang, ein breites Schulangebot trotz zurückgehender Schülerzahlen aufrechtzuerhalten, ist eine relevante Frage, denn Veränderungen in der Schulinfrastruktur und etwaige Ausdünnungen von Schulangeboten haben Auswirkungen auf den Zugang zu Bildungsangeboten und damit auch auf Bildungschancen. Kurze Wege zur nächsten Schule sind bedeutsam für den regionalen und sozial gleichwertigen Zugang zu allen Bildungsabschlüssen. (vgl. Ditton, 2008; Berkemeyer, Bos, Manitius, Hermstein, Bonitz \& Semper, 2014). ${ }^{3}$

Basis unserer Analyse bildeten die Schulverzeichnisse der ostdeutschen Bundesländer der Schuljahre 1992/93 bis 2015/16. Die darin enthaltenen Informationen zu den Adressen der Schulstandorte verknüpften wir zunächst mit Geokoordinaten, die wir dann via Open-Street-Map für jedes Jahr kartierten. Mit Hilfe dieser Web-Anwendung konnte die Entwicklung der Schulstandorte (nach Bildungsgang getrennt) nachverfolgt werden. Das Ergebnis haben wir in einer interaktiven Karte festgehalten (verfügbar unter: https://schulenkarte.wzb.eu; Zugriff am 06.04.2019). Mit dieser Karte und den zugehörigen Berechnungen und Grafiken kann die Entwicklung der Schulangebotsdichte in den ostdeutschen Bundesländern analysiert und dabei die Rolle der privaten Schulen überprüft werden. Wir empfehlen daher, bei der Lektüre dieses Beitrags die Karte und die jeweils genannten Online-Graphiken zu berücksichtigen. Während mittlerweile verschiedene Geo-gestützte Karten von Schulen in Deutschland existieren, ${ }^{4}$ stellt unsere Arbeit die erste dar, die Veränderungen von Schulstandorten grafisch im Zeitverlauf darstellt.

\section{Daten und Operationalisierung}

Mit dem Menüpunkt „Karte“ kann mithilfe eines Reglers auf der linken Seite die Veränderung der Schullandschaft von 1992/93 bis 2015/16 nachvollzogen werden. Wir empfehlen, die Bundesländer separat zu betrachten. Die öffentlichen Schulen sind in blauer Farbe dargestellt, und die privaten Schulen haben wir je nach Trägerschaft bzw. pädagogischem Konzept in kirchliche Schulen, Waldorfschulen und sonstige Privatschulen unterteilt und sie unterschiedlich farblich hervorgehoben. Mit dem Reiter rechts unten können die verschiedenen Schulformen (Schulen mit einem Grundschulteil, Schulen mit bzw. ohne gymnasiale Oberstufe) ausgewählt werden. Unter „Anzeige“ (rechts oben) kann gewählt werden, ob jeweils alle Schulen, die Schulcluster oder die Schulen mit zugehörigem Voronoi-Diagramm ${ }^{5}$ angezeigt werden sollen. Links oben können mit dem Menüpunkt „Begleitgraphiken“ die verschie-

3 Ein ausführliches WZB-Discussion-Paper beschreibt die theoretische Einbettung sowie Vorgehensweise der Analyse und nennt die jeweiligen schulrechtlichen Quellen (Helbig, Konrad \& Nikolai, 2018).

4 Siehe z. B. https://jedeschule.de/schulen/; Zugriff am 06.04.2019.

5 Zur Definition von Voronoi-Diagrammen siehe https://de.wikipedia.org/wiki/Voronoi-Dia gramm; Zugriff am 21.03.2019. 
denen Abbildungen angeklickt werden, auf die wir uns im vorliegenden Beitrag beziehen und die wir mit den jeweiligen Nummern nennen.

Die Schulformen in den Bundesländern sind relativ schwer miteinander vergleichbar. Nicht einmal innerhalb eines Bundeslandes ist anhand des Schulnamens erkennbar, welche Schulform sich tatsächlich dahinter verbirgt. So hatten die meisten Gesamtschulen in Brandenburg nie eine gymnasiale Oberstufe. Gleiches gilt für die stark wachsenden Gemeinschaftsschulen in Thüringen. Auch wenn mit diesen Schulformen eine andere pädagogische Grundhaltung einhergeht, eignen sich die Schulformbezeichnungen nicht für einen Bundesländervergleich über die Zeit. Das einzige sinnvolle Unterscheidungsmerkmal zwischen den weiterführenden Schulen ist, ob es an einer Schule eine gymnasiale Oberstufe gibt. Anhand der Schulverzeichnisse der Länder (bzw. auf Nachfrage in Thüringen) konnten wir für jede integrierte Schule (Gesamt- und Gemeinschaftsschulen) ermitteln, ob es eine gymnasiale Oberstufe an den jeweiligen Schulen gibt. Die weiterführenden Schulen haben wir daher unterteilt in solche mit bzw. ohne gymnasiale Oberstufe. Die integrierten Schulen mit einer gymnasialen Oberstufe zählen wir insoweit „doppelt“, da sie für die Schulen mit und ohne gymnasiale Oberstufe die Dichte des Schulangebots verbessern. Die Gemeinschaftsschulen (vor allem Thüringen), Gesamtschulen (Brandenburg und Mecklenburg-Vorpommern) und Gymnasien (nur Mecklenburg-Vorpommern) zählen wir zudem zu den Grundschulen, wenn sie die Klassen 1 bis 4 beinhalten. In einzelnen Fällen haben wir also Schulen, die in allen drei Kategorien gezählt werden.

Bei den „Schulen mit gymnasialer Oberstufe“ haben wir für alle Schulen festgehalten, ob diese auch tatsächlich eine solche haben. Dies trifft neben den Gymnasien auch auf die Gesamtschulen, einige Gemeinschaftsschulen in Thüringen und die Waldorfschulen zu. Als Schulen „ohne gymnasiale Oberstufe“ haben wir alle NichtGymnasien im Sekundarschulbereich kodiert. Dies bedeutet auch, dass eine Gesamtschule mit gymnasialer Oberstufe oder eine Waldorfschule zweifach auftaucht, bei den Schulen mit und ohne gymnasiale Oberstufe. Hier sind auch die anderen Bildungsabschlüsse neben dem Abitur ein „reguläres Bildungsziel“. Bei der Schulform "Grundschule“ haben wir neben den regulären Grundschulen auch die Waldorfschulen und die Gemeinschaftsschulen in Thüringen codiert.

Insgesamt finden sich in der Schulkarte knapp 140.000 einzelne Datenpunkte. Wird die einzelne Schule angeklickt, so werden für die Datenpunkte der Name und die Adresse der Schule angezeigt. Zudem wird die Entfernung zur nächstgelegenen gleichartigen Schule im gleichen Bundesland angegeben. 
Entwicklung der Schulinfrastruktur in Ostdeutschland und die Rolle privater Schulen |

\section{Methodisches Vorgehen}

Die grafische Darstellung aller Schulen in den ostdeutschen Bundesländern erlaubt es erstens, deskriptiv zu beobachten, wie sich die Zahl der Schulstandorte und -formen entwickelt hat und wo private Schulen entstanden sind. Allerdings ist es auf diese Weise relativ schwierig, präzise Angaben darüber $\mathrm{zu}$ machen, wie sich die Erreichbarkeit von Schulen in einem Bundesland entwickelt hat. Hierfür sind zweitens Kennziffern nötig, die es erlauben, im Zeitverlauf $\mathrm{zu}$ beobachten, wie sich ein Schulnetz verändert hat: So können mit der Erzeugung von Schulclustern die Clusterzu-Cluster-Distanzen und für jeden Schulstandort mit einer dazugehörigen VoronoiRegion ein theoretischer Einzugsbereich jeder Schule berechnet werden.

Das Clustering bildeten wir auf Basis der Luftliniendistanzen zwischen den Schulen, und die Cluster erzeugten wir über ein Distanzkriterium, d.h., innerhalb eines Clusters durfte eine Schule eine bestimmte Maximaldistanz zu jeder anderen Schule dieses Clusters nicht überschreiten. Als Maximaldistanz innerhalb der Cluster haben wir für den Primarbereich einen Abstand von drei Kilometern (km) festgelegt und im Sekundarbereich von fünf Kilometern. Dadurch konnten auch „Einzelcluster“ entstehen, also einzelne Schulen, die selbst ein Cluster darstellen, da ihre nächste Schule weiter entfernt ist, als per Maximaldistanz erlaubt. Die gewählten Distanzkriterien orientieren sich ungefähr daran, was zumutbare Entfernungen sind, die Kinder im jeweiligen Alter zu Fuß bzw. in den weiterführenden Schulen mit dem Fahrrad zurücklegen könnten. Bei einer Distanz von $3 \mathrm{~km}$ zwischen zwei Grundschulen sollte die Entfernung für die Grundschüler*innen, die zwischen diesen Schulen wohnen, $1,5 \mathrm{~km}$ nicht überschreiten. Bei den weiterführenden Schulen wären es entsprechend $2,5 \mathrm{~km}$. Dies trifft natürlich nur dann zu, wenn die Schüler*innen relativ genau zwischen beiden Schulen wohnen. Im Einzelfall wird die Entfernung dementsprechend größer sein.

Ein Clustering von Schulstandorten erlaubte es somit, mehrere, nah beieinander liegende Standorte zu einem Cluster verschmelzen zu lassen. Da es für unsere Analysen nicht wichtig war, wie viele Schulen an einem bestimmten Ort für ein Schulangebot sorgen, sondern $o b$ überhaupt ein solches Angebot an einem Ort existiert (bzw. wie weit die Entfernung dazu ist), erlaubte das Clustering, stabilere Distanzberechnungen vorzunehmen. Schloss eine von insgesamt drei Schulen in einem Cluster, war das für Distanzen zwischen Clustern kaum relevant. Machte allerdings ein „Einzelcluster“ zu, d.h. eine Schule, die allein ein Cluster darstellte, erhöhte sich die Distanz zwischen den umgebenden Clustern signifikant. Inwieweit sich die theoretischen Einzugsbereiche verändert haben, bewerteten wir daran, wie sich die durchschnittlichen Abstände zwischen den Clustern pro Anzahl der Cluster verändert haben. 
In einem Voronoi-Diagramm wurde durch eine Menge von Zentren (in diesem Fall von Schulstandorten) der Raum in mehrere Regionen unterteilt. Jeder zufällige Punkt innerhalb einer solchen Voronoi-Region war näher am Zentrum seiner Region (also hier: Schulstandort) als an irgendeinem anderen Zentrum. So entstand für jede Schule ein künstliches Einzugsgebiet, das eine Fläche beschreibt. Anhand der durchschnittlichen Größe dieser Einzugsgebiete konnten wir die theoretischen Einzugsbereiche der Schulen beschreiben. Abb. 1 zeigt beispielhaft ein VoronoiDiagramm. Die Fläche der äußeren Voronoi-Regionen, hier begrenzt durch gestrichelte Linien, ist dabei theoretisch unendlich groß. Diese Regionen wurden auf die Grenzen der Bundesländer bzw. die Küstenlinie beschränkt. ${ }^{6}$ Wenn Eltern ihre Kinder ggfs. über eine Bundeslandgrenze hinweg zur Schule schicken können, konnten wir das somit nicht mit abbilden.

Abb. 1: Beispiel eines Voronoi-Diagramms mit sieben Punkten

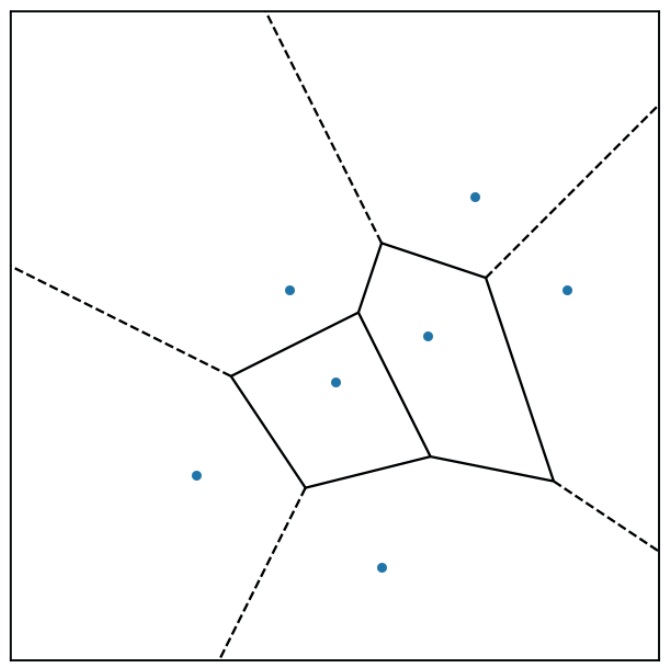

Quelle: eigene Darstellung

Mit beiden Methoden ist ein Vergleich der Bundesländer im Querschnitt nicht zielführend. Denn beide Maße beziehen sich nicht auf die Schulkinder im tatsächlichen Einzugsgebiet, sondern beschreiben nur eine theoretische Raumeinheit. Im Längsschnitt ist der Vergleich anhand der genannten Kennzahlen deutlich sinnvoller. Denn die Ausgangsmessung der Schullandschaft 1992 bildet einen Ausgangszustand ab, der sich auf die damaligen Siedlungsverhältnisse bezieht.

6 Ein Softwarepaket zum Zuschneiden und Abbilden von Voronoi-Regionen für geographische Anwendungen wurde im Rahmen dieser Arbeit entwickelt und wird unter https:// github.com/WZBSocialScienceCenter/geovoronoi frei zur Verfügung gestellt; Zugriff am 06.04.2019. 
Die Betrachtung in Schulclustern und Voronoi-Einzugsgebieten hat eine wichtige Limitation: Sie orientiert sich nicht an der Schülerzahl in einer bestimmten Region. Wenn es im Zeitverlauf zu stark unterschiedlichen räumlichen Schülerzahlentwicklungen kommt, dann wird die Passung zwischen einer Schullandschaft des Jahres 1992 und heutigen Anforderungen schlechter. Für die lokale Schulnetzplanung, die nicht im Fokus dieses Artikels liegt, ist dies eine methodische Herausforderung.

\section{Veränderungen der Schulinfrastruktur in den ostdeutschen Bundesländern}

Nach der Wiedervereinigung 1990 wandelten die fünf ostdeutschen Bundesländer das Schulsystem der DDR, das im Wesentlichen aus der zehnjährigen Polytechnischen Oberschule und der zweijährigen Erweiterten Oberschule bestand, innerhalb weniger Monate um und nahmen unterschiedliche schulstrukturelle Entscheidungen vor (Fuchs, 1997; Nikolai, 2018). Sachsen, Sachsen-Anhalt und Thüringen entschieden sich im Anschluss an eine vierjährige Grundschule für eine zweigliedrige Lösung aus Gymnasien und Schularten mit mehreren Bildungsgängen (mit jeweils den Namen Mittelschule ${ }^{7}$, Sekundarschule oder Regelschule). Brandenburg führte ebenso wie diese drei Bundesländer nicht die Hauptschule als eigenständige Schulform ein, legte sich aber zunächst mit Gymnasium, Gesamtschule und Realschule auf drei Sekundarschulformen fest. Wie in Berlin dauert die Grundschule in Brandenburg sechs Jahre.

Mecklenburg-Vorpommern folgte dem westdeutschen Pfad und führte ein viergliedriges Schulsystem mit Haupt-, Real-, Gesamtschulen und Gymnasien ein. Es gab allerdings nur wenige Hauptschulen in Mecklenburg-Vorpommern (10\% der Schüler*innen in der Sekundarstufe I im Jahr 1993: StBa, 2018a; eigene Berechnungen). In den 1990er-Jahren nahmen die ostdeutschen Bundesländer weitere Veränderungen in der Schulstruktur vor. So wurde die Gesamtschule als eine weitere Schulform in Thüringen 1992/93 und in Sachsen-Anhalt 1997/98 eingeführt. In Thüringen wurde zum Schuljahr 2010/11 auch die Gemeinschaftsschule ermöglicht. Zweigliedrige Schulsysteme wurden in Brandenburg zum Schuljahr 2005/06 und in Mecklenburg-Vorpommern 2006/07 etabliert (Helbig \& Nikolai, 2015).

Für Brandenburg ist festzustellen, dass neben den Gymnasien bis zur Etablierung eines zweigliedrigen Schulsystems vorrangig Gesamtschulen bestanden; Realschulen gab es nur an wenigen Schulstandorten. Dabei hatten viele der Brandenburger Gesamtschulen keine gymnasiale Oberstufe (für eine detaillierte Übersicht vgl. Helbig \& Nikolai, 2015, S. 53 ff.). Ähnlich wie derzeit in Berlin, Hamburg, SchleswigHolstein und dem Saarland gibt es aber an der zweiten Sekundarschulform neben

7 In Sachsen wurde die Mittelschule 2013/14 umbenannt in Oberschule. 
dem Gymnasium nicht an jeder Schule eine gymnasiale Oberstufe (Stand Schuljahr 2017/18). Welche Schule eine gymnasiale Oberstufe hat, ergibt sich in Brandenburg aus dem Schulverzeichnis. In Brandenburg sollten die unterschiedlichen Gesamtschulen miteinander kooperieren und gemeinsame gymnasiale Oberstufen betreiben. Hier geschah dies vornehmlich über die Oberstufenzentren, die wir ebenfalls (nur für Brandenburg) farblich abgestuft in der Karte festgehalten haben.

Nicht nur die Schulstrukturen unterschieden sich zwischen den ostdeutschen Bundesländern, Unterschiede bestanden auch hinsichtlich der Siedlungsdichte (gemessen an Einwohner*innen pro $\mathrm{km}^{2}$ ). $\mathrm{Zu}$ den Bundesländern mit der geringsten Bevölkerungsdichte gehören im Bundesländervergleich Mecklenburg-Vorpommern und Brandenburg. Mit etwas Abstand folgen Sachsen-Anhalt und Thüringen. Sachsen liegt zwar unter dem Bundesdurchschnitt der Bevölkerungsdichte, hat aber eine höhere Siedlungsdichte als etwa Bayern, Rheinland-Pfalz, Schleswig-Holstein oder Niedersachsen. Sachsen-Anhalt, Thüringen und insbesondere Sachsen weisen anders als Brandenburg oder Mecklenburg-Vorpommern eine größere Zahl von Städten und auch Großstädte auf (StBa, 2018b). Aufgrund der z. T. beträchtlichen Unterschiede in der Bevölkerungsdichte stellte der Rückgang der Schülerzahlen die fünf ostdeutschen Bundesländer auch vor unterschiedliche Herausforderungen.

So verringerte sich die Zahl der Schulkinder im Alter von 7 bis 15 Jahren in den ostdeutschen Bundesländern bis 2007 auf 38,8 (Mecklenburg-Vorpommern) bis 48,4 Prozent der Ausgangswerte (Brandenburg) (Abb. 1-web). Auch wenn der Anstieg der Schülerzahlen ab 2007 bis 2015 um 10,6 (Sachsen-Anhalt) bis 22,5 Prozent (Sachsen) im Spiegel des historischen Einbruchs niedrig erscheinen mag, so sind diese Nachfrageveränderungen für die Schulnetz- und Personalplanung eine immense Herausforderung (Kann, 2017). Zudem war der Einbruch bei der Grundschul(Abb. 2-web) und der Sekundarschulpopulation (Abb. 3-web) noch tiefer, und der Tiefpunkt wurde zu unterschiedlichen Zeiten erreicht (2002 bzw. 2007). Schließlich war der Einbruch der Schülerzahlen im Sekundarschulbereich an Gymnasien bzw. Schulen mit gymnasialer Oberstufe weniger stark zu spüren (Abb. 4-web und 5-web).

Während die Schülerschaft in den ostdeutschen Bundesländern insgesamt stark zurückging, erstarkte die Zahl privater Schulen (Abb. 6-web bis 9-web). Ihre Expansion begann in den östlichen Bundesländern dabei erst so richtig, als der Bevölkerungseinbruch seinen Tiefpunkt bereits erreicht hatte. Kaum aufrechtzuerhalten ist die Annahme, dass private Schulen für eine bessere Dichte des Schulangebots im ländlichen Raum gesorgt hätten (Autorengruppe Bildungsberichterstattung, 2018, S. 88) oder diese mit „ihren Angeboten erfolgreich ,eingesprungen“ (Klemm, Hoffmann, Maaz \& Stanat, 2018, S. 18) seien. Neben der allgemeinen Ausdünnung der Schullandschaft zeigt sich, dass sich die privaten Schulen vor allem in den mittleren und größeren Städten bzw. für Brandenburg geballt rund um Berlin angesiedelt 
Entwicklung der Schulinfrastruktur in Ostdeutschland und die Rolle privater Schulen |

haben. ${ }^{8}$ Wie sich die Dichte des Schulangebots in den ostdeutschen Bundesländern darstellt und wie private Schulen dazu beitragen, stellen wir gegliedert nach Schulformen dar.

\subsection{Grundschulen}

Bei den Grundschulen war der Rückgang der Zahl der Schulen (Abb. 10-web) weniger stark als der Einbruch bei den Schülerzahlen (Abb. 2-web). Abbildung 16-web zeigt, wie sich die Anzahl der Schulcluster im Grundschulbereich seit 1992 entwickelt hat. Während die Anzahl der Grundschulen auf 56 (Mecklenburg-Vorpommern) bis 80 Prozent (Brandenburg) ihres Ausgangsniveaus zurückgegangen ist (Abb. 10-web), verringerte sich die Anzahl der Schulcluster auf 63 (Mecklenburg-Vorpommern) bis 86 Prozent. Thüringen verlor dabei bereits ab 1999 nur noch graduell Schulcluster, Sachsen ab 2002 und Brandenburg ab 2006. Demgegenüber ist es in MecklenburgVorpommern 2005 bis $2010 \mathrm{zu}$ einem sehr starken Einbruch der Schulcluster gekommen (ab 2010 stabil), und in Sachsen-Anhalt hält diese Entwicklung seit 2009 an. Die Karte zeigt ab 2010 für Sachsen-Anhalt einen massiven Rückgang der Grundschulstandorte an der Grenze zu Brandenburg und im Norden in der Altmark Sachsen-Anhalts. ${ }^{9}$

Insgesamt bleibt festzuhalten, dass sich der Rückgang von Grundschulen überproportional innerhalb von Grundschulclustern vollzog und dass er weniger stark den Rückgang von Grundschulclustern zur Folge hatte. Auch wenn wir die Entfernungen zwischen den Grundschulclustern heranziehen (Abb. 19-web) ${ }^{10}$ können wir feststellen, dass fast alle ostdeutschen Bundesländer über die Zeit ein enges Grundschulnetz aufrechterhalten konnten, wenn auch mit deutlichen Niveauunterschieden.

Hatten Sachsen, Sachsen-Anhalt und Thüringen 1992 noch eine durchschnittliche Entfernung zwischen den Grundschulclustern von unter $5 \mathrm{~km}$, in Sachsen sogar von unter $4 \mathrm{~km}$, waren die Grundschulcluster in Brandenburg und Mecklenburg-Vorpommern mit durchschnittlich $5,7 \mathrm{~km}$ viel weiter voneinander entfernt. Die Entfernungen zwischen den Grundschulclustern erhöhten sich zwar im Zeitverlauf bis

8 Besonders hoch ist der Anteil von privaten Grundschulen (inklusive Waldorfschulen, die auch mit Klasse 1 beginnen) an allen Grundschulen 2015 in Schwerin (44 Prozent), Rostock (29 Prozent), Greifswald (44 Prozent), Neubrandenburg (25 Prozent) und Potsdam (33 Prozent) (siehe auch Karte).

9 Neben der Betrachtung der Anzahl der Schulen wäre es auch interessant gewesen, die Schüleranteile in den jeweiligen Jahrgangsstufen zu betrachten. Jedoch bieten Schullisten nicht diese detaillierten Informationen, und zudem ist es für uns noch eine offene methodische Frage, wie Zahlen zur Jahrgangsstärke in einem Raum darstellbar sein können.

10 Ebenso haben wir dargestellt, wie sich die einzelnen Schulcluster nach verschiedenen Entfernungskategorien in den Jahren 1995, 2005 und 2015 verteilten, jeweils für die Grundschulen (Abb. 20.1-web bis 20.5-web) und für die Sekundarschulen mit und ohne gymnasiale Oberstufe (Abb. 22.1-web bis 22.5-web bzw. Abb. 24.1-web bis 24.5-web). 
2015 in fast allen ostdeutschen Bundesländern zwischen 0,63 km und 0,85 km, doch nur in Mecklenburg-Vorpommern nahmen die Entfernungen auf mehr als $7 \mathrm{~km} \mathrm{zu}$. In diesem Bundesland hat sich das lokale Grundschulangebot gegenüber 1992 gemessen an den Entfernungen zwischen den Grundschulclustern deutlich verschlechtert. Betrachten wir die Voronoi-Flächen, also die künstlichen Schuleinzugsgebiete (Abb. 25.1-web bis 25.5-web), dann zeigt sich vor allem ein Rückgang der Grundschulen mit einem Einzugsgebiet von unter 50 Quadratkilometern $\left(\mathrm{km}^{2}\right)$. Gleichzeitig ist die Anzahl von Grundschulen mit Einzugsgebieten von über $50 \mathrm{~km}^{2}$ angestiegen. Sowohl in Brandenburg und Sachsen-Anhalt als auch in Mecklenburg-Vorpommern gibt es mittlerweile Grundschulen, die ein Einzugsgebiet von über $300 \mathrm{~km}^{2}$ haben.

Für alle ostdeutschen Bundesländer können wir festhalten, dass private Grundschulen wenig zur Schulangebotsdichte beigetragen haben. Sie entstanden überwiegend im städtischen Raum, sie befinden sich nur selten weiter als $6 \mathrm{~km}$ von der nächsten öffentlichen Schule entfernt (Abb. 13.1-web bis 13.5-web), sie verringern kaum die durchschnittliche Entfernung zwischen den einzelnen Schulclustern (Abb. 19-web bis 20.5-web.), und sie decken vorrangig Schuleinzugsgebiete von unter $50 \mathrm{~km}^{2} \mathrm{ab}$ (Abb. 25.1-web bis 25.5-web). Einzig in Mecklenburg-Vorpommern und Brandenburg gibt es vereinzelt private Grundschulen im ländlichen Raum, die die Erreichbarkeit von Grundschulen verbessern.

\subsection{Schulpolitik und Dichte des Grundschulangebots}

Brandenburg, Thüringen, Sachsen und teilweise Sachsen-Anhalt konnten stärker an bestehenden Grundschulclustern festhalten (Abb. 16-web) als Mecklenburg-Vorpommern, und dementsprechend konnten sie auch eine bessere räumliche Dichte des Grundschulangebots erhalten (Abb. 19-web). Eine wichtige Rolle hatten dabei schulrechtliche Regelungen, die es ermöglichten, Grundschulstandorte trotz zurückgehender Schülerzahlen bestehen zu lassen.

Im Untersuchungszeitraum erlaubte Brandenburg, dass Schulen an mehreren Standorten gemeinsam als eine Schule geführt werden und Grundschulen auch jahrgangsübergreifend unterrichten können. Zudem waren einzügige Grundschulen möglich, und die Mindestschülerzahl an Grundschulen betrug 15 Schulkinder pro Klasse. Ähnlich niedrig war die Mindestschülerzahl in Sachsen. In Thüringen wurden entsprechende Richtlinien zur Mindestzügigkeit vom Kultusministerium nie erlassen (Kann, 2017, S. 113), wodurch es in Thüringen keinen „demografischen Automatismus“ gibt, der zur Grundschulschließung führt. Im Bundesländervergleich (auch mit den westdeutschen Bundesländern) ist Thüringen das einzige Bundesland, das noch keine Vorgaben zur Klassenbildung für den Grund- oder auch den Sekundarschulbereich macht (KMK, 2018). Jahrgangsübergreifender Unterricht an Grundschulen war zwar auch in Mecklenburg-Vorpommern vorgesehen, doch wurden die 
Mindestschülerzahlen sukzessive auf 20 Schulkinder in der 1. Jahrgangsstufe erhöht (Kann, 2017). Die weniger rigiden Regelungen für den Betrieb von Grundschulen könnten in Brandenburg, Sachsen und Thüringen zu einer höheren Dichte des Grundschulangebots als in Mecklenburg-Vorpommern geführt haben. In SachsenAnhalt gelang dies zuletzt nicht mehr, obwohl auch hier eine weniger rigide Mindestzügigkeit von 15 Schulkindern bzw. 13 in dünnbesiedelten Regionen (in Magdeburg, Stadt Halle (Saale) und Stadt Dessau-Roßlau 20 Schulkinder) und auch die Einzügigkeit von Grundschulen ermöglicht wurden.

\subsection{Sekundarschulen}

Ähnlich wie bei den Grundschulen wurde die Dichte des Angebots der Sekundarschulen untersucht, getrennt für die Sekundarschulen ohne gymnasiale Oberstufe (Abb. 11-web, 17-web, 21-web, 22.1-web bis 22.5-web, 26.1-web bis 25.5-web) und mit gymnasialer Oberstufe (Abb. 12-web, 18-web, 23-web, 24.1-web bis 24.5-web, 27.1-web bis 27.5-web).

Bei den Schulen ohne gymnasiale Oberstufe wird ein Rückgang der Schulzahl auf gut 30 bis knapp 60 Prozent der Ausgangsschulzahl deutlich (Abb. 11-web) der Einbruch der Schulzahlen ist einschneidend. In Thüringen und Sachsen blieben über 80 Prozent aller Schulcluster bestehen, während in den anderen drei Bundesländern nur 50 bis 60 Prozent erhalten blieben (Abb. 17-web). Die Schließung der Schulstandorte konzentrierte sich in Thüringen und Sachsen eher auf eine Ausdünnung innerhalb von Schulclustern als auf eine Schließung von Clustern. Dadurch blieb die Entfernung zwischen den einzelnen Schulclustern in Thüringen und etwas weniger in Sachsen relativ stabil. Zwischen 1992 und 2015 stieg der Abstand der Schulcluster in Thüringen durchschnittlich um weniger als $1 \mathrm{~km}$ an. In Sachsen war der Anstieg mit 1,3 km etwas höher. In Brandenburg, Sachsen-Anhalt und Mecklenburg-Vorpommern erhöhten sich die Abstände zwischen den einzelnen Schulclustern durchschnittlich um 2 bis 2,7 km (Abb. 21-web). Ein ähnliches Bild lässt sich aus den Schuleinzugsgebieten der Voronoi-Diagramme ableiten (Abb. 26.1web bis 26.5-web).

Es kam in allen Bundesländern zu einem Rückgang der Schulen ohne gymnasiale Oberstufe mit einem Einzugsgebiet unter $50 \mathrm{~km}^{2}$. Gleichzeitig blieb die Anzahl von Schulen mit Einzugsgebieten über $50 \mathrm{~km}^{2}$ konstant bis steigend. Sowohl in Brandenburg als auch in Sachsen-Anhalt gibt es mittlerweile Schulen ohne gymnasiale Oberstufe, die ein Einzugsgebiet von über $500 \mathrm{~km}^{2}$ haben. Eigentlich könnten die Sekundarschulen ohne gymnasiale Oberstufe im Sekundarbereich eine „Grundversorgung" bereitstellen, doch zumindest in Sachsen-Anhalt, Brandenburg und Mecklenburg-Vorpommern ist in diesem Schulbereich eine Ausdünnung zu beobachten. Der Privatschulbereich trug relativ wenig dazu bei, dieser Entwicklung et- 
was entgegenzusetzen bzw. für eine höhere Dichte des Schulangebots zu sorgen (siehe Abb. 14.1-web bis 14.5-web, 21-web, 22.1-web bis 22.5-web, 26.1-web bis 26.5-web).

Bei den Schulformen mit gymnasialer Oberstufe ist bereits 1992 ein relativ ausgedünntes Bild festzustellen. Die Abstände zwischen einzelnen öffentlichen Gymnasien lagen bereits damals oft über $15 \mathrm{~km}$. Im Norden Sachsen-Anhalts und Brandenburgs sowie in Mecklenburg-Vorpommern ist ein Abstand zwischen diesen Schulen von über $25 \mathrm{~km}$ keine Ausnahme. Insgesamt ist es zudem zu einer Ausdünnung gekommen, die einige Regionen besonders betroffen hat (den Westen Brandenburgs und die Altmark in Sachsen-Anhalt); aber insgesamt ist das Netz von Schulen mit gymnasialer Oberstufe trotz des Bevölkerungsrückgangs relativ stabil geblieben. Die Standorte der Schulen mit gymnasialer Oberstufe sind trotz massiven Rückgangs der Zahl der Schüler*innen zumindest in Thüringen, Sachsen und Mecklenburg-Vorpommern fast alle erhalten geblieben. 2015 existierten dort 90 bis 94 Prozent der Schulcluster von 1992 (Abb. 18-web). Auch die Entfernung zwischen den Schulclustern blieb in Thüringen, Sachsen-Anhalt, Sachsen und Mecklenburg-Vorpommern gemessen an allen Schulen (privat und öffentlich) relativ konstant im Zeitverlauf (Abb. 23-web). Dies gelang aber nur in Thüringen aus dem öffentlichen Schulnetz heraus.

Abgesehen von Thüringen wäre in den anderen ostdeutschen Bundesländern ohne die Gründung privater Schulen mit gymnasialer Oberstufe die Abdeckung wesentlich schlechter gewesen. In Sachsen, Sachsen-Anhalt und Mecklenburg-Vorpommern wurde die Schließung öffentlicher Gymnasien durch private Gymnasien kompensiert, die die alten öffentlichen Gymnasialstandorte übernahmen (siehe Karte).

Im Gegensatz zu Sachsen, Mecklenburg-Vorpommern und Thüringen sank die Zahl der Schulcluster in Brandenburg und Sachsen-Anhalt auf 80 bzw. 72 Prozent (Abb. 18-web). In Brandenburg ist dies insoweit überraschend, als hier die Schulzahl mit gymnasialer Oberstufe konstant blieb (Abb. 12-web). Private Gymnasien trugen in Brandenburg wenig zur Erreichbarkeit bei, weil diese sich eher im Berliner Umland befanden, wo sie keine eigenen Schulcluster darstellten. Außerhalb des Berliner "Speckgürtels" verschwanden Schulcluster mit der Schließung örtlicher Gymnasien; die Voronoi-Einzugsgebiete der Gymnasien verdeutlichen dies (Abb. 27.1-web bis 27.5-web). Anders als bei den anderen Schulformen kam es zu geringen Verschiebungen der Einzugsgebiete. In allen fünf Bundesländern gibt es mittlerweile Schulen mit gymnasialer Oberstufe, die ein Einzugsgebiet von 500 bis $1.000 \mathrm{~km}^{2}$ aufweisen, in Brandenburg und Sachsen-Anhalt sogar Gymnasien mit einem Einzugsgebiet von über $1.000 \mathrm{~km}^{2}$.

Bei den Schulen mit gymnasialer Oberstufe spielen die privaten Schulen eine deutlich wichtigere Rolle für die Dichte des Schulangebots, wobei eine Vielzahl privater Gymnasien sich in den größeren Städten befindet. So ist der Anstieg der Zahl der Gymnasien ab 2007 auf die Gründung privater Gymnasien zurückzuführen (Abb. 12- 
web); diese befinden sich in nennenswerter Anzahl über $10 \mathrm{~km}$ von der nächsten öffentlichen Schule entfernt (Abb. 15.1-web bis 15.5-web) und tragen (außer in Thüringen) dazu bei, dass die Entfernung zwischen den Schulclustern ab 2004 nicht zu-, sondern teilweise sogar abnimmt (Abb. 23-web).

\subsection{Schulpolitik und Dichte des Sekundarschulangebots}

Auch im Sekundarschulbereich gibt es schulpolitische Regelungen, die die Dichte des Schulangebots beeinflussen. Im Bereich der Schulen ohne gymnasiale Oberstufe ist auf die Orientierungsstufen in Sachsen-Anhalt (1997/98 bis 2002/03) und in Mecklenburg-Vorpommern ab 2006/07 hinzuweisen. In beiden Ländern gingen bzw. gehen zunächst alle Schulkinder in den Klassen 5 und 6 auf die nicht-gymnasialen Schulformen und wechselten bzw. wechseln erst ab Klasse 7 auf die Gymnasien (Helbig \& Nikolai, 2015, S. 83). Diese Schulreform könnte auch als eine Stützmaßnahme für die nicht-gymnasiale Schulform verstanden werden. Allerdings ist mit den vorliegenden Daten nicht nachweisbar, dass die Orientierungsstufen in beiden Ländern die Dichte des Schulangebots bei den nicht-gymnasialen Schulformen erhöht hätten.

Die Schulangebotsdichte wird ebenso durch die Mindestschülerzahl beeinflusst. Sachsen macht relativ rigide Vorgaben, denn Gymnasien müssen mindestens dreizügig sein, und die Einrichtung von Gesamtschulen ist bis heute schulrechtlich ausgeschlossen (Helbig \& Nikolai, 2015). Dies mag dazu geführt haben, dass es in Sachsen vermehrt zu Schließungen von öffentlichen zu Gunsten von privaten Gymnasien kam. Solch rigide Vorschriften könnten aber auch Kettenreaktionen auslösen, indem durch die Etablierung neuer privater Gymnasien den öffentlichen Gymnasien die bestandswahrende Schülerzahl entzogen wird.

Die Ermöglichung integrierter Schulformen kann eine zentrale Stellschraube sein, um Schulstandorte aller Schulformen zu sichern. Während Sachsen Gesamtschulen nicht zulässt, nutzen z.B. in Mecklenburg-Vorpommern private Anbieter diese Möglichkeit, und vornehmlich an der Ostseeküste haben sich private Gesamtschulen etabliert, wo öffentliche Gymnasien eine „Lücke“ ließen.

Den Weg über das staatliche Schulsystem geht Thüringen, das zum Schuljahr 2011/12 die Gemeinschaftsschule als eine Regelschulform einführte. Sie gilt dabei als eine „schulstrukturelle Alternative“ zur "Aufrechterhaltung der Schulstandorte" und zur „Gewährung eines einheitlichen Bildungsangebots auch im ländlichen Raum“ (TMBJS, 2016, o. S.). Anders als die integrierten Gesamtschulen integrieren die Gemeinschaftsschulen auch die Grundschulen. Gymnasiale Oberstufen existieren bisher nur an wenigen der Thüringer Gemeinschaftsschulen. 


\section{Fazit}

Mit der Analyse der Schulstandortentwicklung in den ostdeutschen Bundesländern konnten wir zunächst veranschaulichen, wie einfachste Angaben der amtlichen Statistik (in unserem Fall Schulverzeichnisse) mit multimedialen Mitteln aufbereitet und analysiert werden können. Zudem konnten wir zeigen, dass es angesichts der enormen demografischen Herausforderungen der letzten 25 Jahre in den ostdeutschen Bundesländern relativ gut gelungen ist, zumindest das Grundschulangebot in der Fläche abzusichern (mit Abstrichen in Mecklenburg-Vorpommern). Bei den Schulen ohne gymnasiale Oberstufe ist dies nur für Thüringen und teilweise für Sachsen zu attestieren. Die privaten Schulen spielten für die Erreichbarkeit von Grund- und Sekundarschulen ohne gymnasiale Oberstufe eine marginale Rolle. In nur wenigen Fällen konnten sie die Angebotsdichte verbessern.

Bei den Schulen mit gymnasialer Oberstufe zeigt sich ein anderes Bild. Hier kam es in allen ostdeutschen Bundesländern zu einem geringeren Rückgang der Dichte des Schulangebots. Besonders in Sachsen und Mecklenburg-Vorpommern sorgen private Anbieter zudem für eine bessere räumliche Erreichbarkeit von Schulstandorten. Nichtdestotrotz gibt es mittlerweile in allen ostdeutschen Bundesländern Schulen mit gymnasialer Oberstufe, die ein Einzugsgebiet von 500 bis $1.000 \mathrm{~km}^{2}$ und sogar von über $1.000 \mathrm{~km}^{2}$ haben. Welche Auswirkungen solch große Einzugsgebiete auf Bildungschancen haben, bleibt zu überprüfen. Beiträge aus Sixt, Bayer \& Müller (2018) weisen jedoch darauf hin, dass weite Schulentfernungen insbesondere sozial benachteiligte Schulkinder von einem Gymnasialbesuch bzw. Besuch einer Schule mit gymnasialer Oberstufe abhalten können.

Privatschulen spielen im Osten bei der räumlichen Grundversorgung von Bildungseinrichtungen (Grundschulen und Schulen ohne gymnasiale Oberstufe) eine untergeordnete Rolle. Private Schulen sind auch dort kaum entstanden, wo öffentliche Schulen schließen mussten; vielmehr befinden sie sich vor allem in den Städten. Die hohe Anzahl privater Grundschulen in Schwerin, Rostock oder Potsdam ist insoweit bedenklich, als im Grundgesetz Art. 7 Abs. 5 die Hürden zur Genehmigung privater Schulen hoch angesetzt sind. Historisch ist diese Regelung als Absage an gymnasiale Vorbereitungsschulen zu sehen und folgt dem Ideal einer gemeinsamen Primarstufe für Schulkinder aller Schichten. Jedoch werden sowohl die Umsetzung der Grundgesetzvorgaben als auch das Sonderungsverbot nach Art. 7 Abs. 4 wenig kontrolliert (Wrase \& Helbig, 2016).

Unsere Analyse fokussierte auf die räumliche Erreichbarkeit und die Dichte des Schulangebots als eine räumliche Kategorie. Nicht berücksichtigen konnten wir dabei die jeweiligen Entscheidungsprozesse und auch Abwägungsprozesse schulpolitischer Akteure in Landesministerien und in den Kommunen bei der Schul- 
Entwicklung der Schulinfrastruktur in Ostdeutschland und die Rolle privater Schulen |

entwicklungsplanung. Mitunter treffen Landesministerien vor dem Hintergrund der Ressourcensteuerung unliebsame Entscheidungen, die sich in den Kommunen nur schwer durchsetzen lassen oder mit Kompromissen und Reibungsverlusten einhergehen.

Der Beitrag weist zudem darauf hin, dass flexible Regelungen zu Mindestschülerzahlen oder die Gründung integrierter Schulformen eine höhere Dichte des Schulangebots gewährleisten können. Nicht aufzeigen können wir, wo es schon Schulen mit mehreren Schulstandorten gibt, die als Kooperationsschulen bereits heute zusammenarbeiten (siehe TMBJS, 2017). In Thüringen existieren im Schuljahr 2018/19 zwar 83 Schulen mit mehreren Schulgebäuden (Datenlieferung des Thüringer Kultusministeriums); allerdings handelt es sich nur in 20 Fällen um Schulen, die in unterschiedlichen Gemeinden liegen. ${ }^{11}$

Die Beispiele aus Ostdeutschland zeigen, dass integrierte Schulformen und auch Kooperationen zwischen Schulen (z.B. gemeinsame Oberstufen oder auch Zweigstellen) notwendig sind, um Schulstandorte aufrechterhalten zu können. Nicht nur in Ostdeutschland ist zur Schulstandortsicherung gerade im ländlichen Raum Pragmatismus von Nöten, der auch die Gymnasien einbeziehen sollte und auch in Bundesländern notwendig wäre, die entweder keine Schulformenintegrationen (z.B. Gesamt- oder Gemeinschaftsschule) vornehmen und/oder an den nicht-gymnasialen Schulformen keine Oberstufe ermöglichen.

\section{Literatur und Internetquellen}

Autorengruppe Bildungsberichterstattung (2018). Bildung in Deutschland 2018. Bielefeld: Bertelsmann.

Berkemeyer, N., Bos, W., Manitius, V., Hermstein, B., Bonitz, M., \& Semper, I. (2014). Chancenspiegel 2014. Gütersloh: Bertelsmann Stiftung.

Ditton, H. (2008). Schule und sozial-regionale Ungleichheit. In W. Helsper \& J. Böhme (Hrsg.), Handbuch der Schulforschung (S. 631-649). Wiesbaden: VS. https://doi.org/ 10.1007/978-3-531-91095-6_25

Fickermann, D., Schulzeck, U., \& Weishaupt, H. (2000). Zur Effizienz regionaler Standortsysteme am Beispiel von Mecklenburg-Vorpommern. In M. Weiß \& H. Weishaupt (Hrsg.), Bildungsökonomie und Neue Steuerung (S. 169-202). Frankfurt a. M. et al.: Peter Lang.

Fuchs, H.-W. (1997). Bildung und Wissenschaft seit der Wende. Opladen: Leske + Budrich.

Helbig, M., Konrad, M., \& Nikolai, R. (2018). Die Schulinfrastruktur in Ostdeutschland (WZB Discussion Paper P 2018-006). Berlin: WZB. Zugriff am 21.03.2019. Verfügbar unter: https://bibliothek.wzb.eu/pdf/2018/p18-006.pdf.

Helbig, M., \& Nikolai, R. (2015). Die Unvergleichbaren. Bad Heilbrunn: Klinkhardt.

11 Für die anderen Bundesländer liegt uns die Aufstellung von Schulen mit mehreren Schulstandorten nicht vor. Auch hier sind derartige Modelle zu erwarten. 
Kann, C. (2017). Schulschließungen und Umbau von Schulstandorten. Wiesbaden: Springer VS. https://doi.org/10.1007/978-3-658-18269-4

Klemm, K., Hoffmann, L., Maaz, K., \& Stanat, P. (2018). Privatschulen in Deutschland. Berlin: Friedrich-Ebert-Stiftung.

KMK (Sekretariat der Ständigen Konferenz der Kultusminister der Länder in der Bundesrepublik Deutschland) (2018). Vorgaben für die Klassenbildung. Schuljahr 2018/2019. Bonn: KMK.

Koinzer, T., \& Mayer, T. (2015). Private Schulen. Zeitschrift für Grundschulforschung, 8 (2), $28-41$.

Nikolai, R. (2018). Schulpolitik im Wandel. Berlin et al.: Peter Lang.

Sixt, M., Bayer, M., \& Müller, D. (Hrsg.). (2018). Bildungsentscheidungen und lokales Angebot. Münster et al.: Waxmann.

StBa (Statistisches Bundesamt) (2018a). Bildung und Kultur. Allgemeinbildende Schulen. Schuljahr 2017/2018. Fachserie 11. Wiesbaden: StBa.

StBa (Statistisches Bundesamt) (2018b). Bundesländer mit Hauptstädten nach Fläche, Bevölkerung und Bevölkerungsdichte. Wiesbaden: StBa.

TMBJS (Thüringer Ministerium für Bildung, Jugend und Sport) (2016). Die Thüringer Gemeinschaftsschule (Flyer). Erfurt: TMBJS. Zugriff am 21.03.2019. Verfügbar unter: https://tinyurl.com/yddq4wva.

TMBJS (Thüringer Ministerium für Bildung, Jugend und Sport) (2017). Zukunft Schule. Erfurt: TMBJS.

Walde, A. (2019). Schulpolitik in Städten mit Schülerrückgang. Wiesbaden: Springer VS.

Weishaupt, H. (2018). Bildung und Region. In R. Tippelt \& B. Schmidt-Hertha (Hrsg.), Handbuch Bildungsforschung (S. 271-286). Wiesbaden: VS. https://doi.org/10.1007/9783-531-19981-8_10

Wrase, M., \& Helbig, M. (2016). Das missachtete Verfassungsgebot. Neue Zeitschrift für Verwaltungsrecht, 35 (22), 1591-1598.

Marcel Helbig, geb. 1980, Professor für „Bildung und soziale Ungleichheit“ an der Universität Erfurt und am Wissenschaftszentrum Berlin für Sozialforschung.

E-Mail: marcel.helbig@uni-erfurt.de

Anschrift: Universität Erfurt, Erziehungswissenschaftliche Fakultät, Postfach 900 221, 99105 Erfurt

Markus Konrad, geb. 1987, Data Scientist im Wissenschaftszentrum Berlin für Sozialforschung.

E-Mail: markus.konrad@wzb.eu

Anschrift: Wissenschaftszentrum Berlin für Sozialforschung, Reichpietschufer 50, 10785 Berlin

Rita Nikolai, geb. 1977, Heisenberg-Stipendiatin der Deutschen Forschungsgemeinschaft (DFG) am Institut für Erziehungswissenschaften der Humboldt-Universität zu Berlin.

E-Mail: rita.nikolai@hu-berlin.de

Anschrift: Humboldt-Universität zu Berlin, Institut für Erziehungswissenschaften, Geschwister-Scholl-Str. 7, 10099 Berlin 